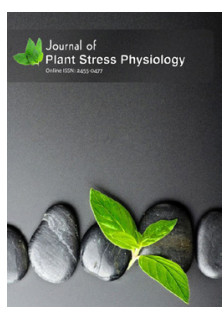

ISSN: $2455^{-0} 0477$

\title{
Fatty acid desaturases modulated octadecanoid pathway in Sesame
}

\author{
Muthulakshmi Chellamuthu, Reshma Sanal, Selvi Subramanian* \\ Department of Biotechnology, PSG College of Technology, Coimbatore, Tamil Nadu, India
}

\begin{abstract}
Omega 3 fatty acid desaturases are involved in the production of $\alpha$-linolenic acid (LNA) an essential omega 3 fatty acid, which is present in only traces in sesame seeds. LNA is the precursor for jasmonic acid which is the end product of octadecanoid pathway. This study was undertaken to analyze the key components of octadecanoid pathway and its relationship with fatty acid content in sesame. Fatty acid desaturation and membrane fluidity are modulated differentially in various stresses. Sesame seedlings were subjected to transient stress to analyse the octadecanoid pathway and its impact on fatty acid desaturation. The mRNA levels of omega 3 desaturases and LNA content were higher in cold stressed sesame seedlings than heat, drought and salinity stresses. The LOX activity and MDA content were higher in heat stressed sesame seedlings. Jasmonic acid content was higher in salinity stressed seedlings while abscisic acid registered the highest in drought stressed seedlings. Chloroplast fatty acid desaturase genes expression was found to increase the LNA content in cold stressed seedlings. The level of membrane damage measured by lipid peroxidation in terms of LOX activity and MDA content were found to be minimal in cold stressed seedling. This suggests a possible role of LNA in membrane fluidity and cold acclimation in sesame. A synergistic role of JA and ABA is also suspected in abiotic stress tolerance in sesame.
\end{abstract}

*Corresponding Author:

Selvi Subramanian

Email: selvi@bio.psgtech.ac.in

KEYWORDS: Sesamum indicum, abscisic acid, jasmonic acid, lipoxygenase, malondialdehyde, linolenic acid

\section{INTRODUCTION}

Sesame (Sesamum indicum L.) is a major oil seed crop characterized with a maximum of $59.8 \%$ oil content and the oil is known to be resistant to oxidation and rancidity [1]. It is one of the first oil seeds recognized by humankind and more than $80 \%$ of the total fatty acids in the sesame oil is composed of oleic (Cl8:1) and linoleic (LA) (Cl8:2) acids and trace amounts of linolenic acid (LNA) (Cl8:3) [2]. High levels of mono unsaturated (UFA) and polyunsaturated fatty acids (PUFAs) increase the quality of oil and has positive effects in reducing blood cholesterol, high blood pressure and thus, play an important role in preventing atherosclerosis, heart diseases and cancers [3]. Sesame oil contains sesamin and sesamolin lignans in its non-glycerol fraction which are known to play an important role in the oxidative stability and antioxidant activity [4].

A series of fatty acid desaturases perform the desaturation reaction of dienoic acids to trienoic acids. Besides, desaturases play vital role in plant development, seed oil accumulation and stress tolerance such as temperature [5], light [6] pathogen attack, wounding and in hormone regulation $[7,8]$. Lipid composition and degree of fatty acid desaturation affect the membrane structure and fluidity. Several reports indicate the effect of environmental stress over the lipid composition [9].Omega 3 fatty acid desaturases are enzymes responsible for formation of trienoic fatty acids. There are three distinct membrane bound omega 3 desaturases, FAD3 located in endoplasmic reticulum, FAD7 and FAD8 located in chloroplast. Trienoic fatty acid is the precursor of octadecanoid pathway which ends in jasmonic acid (JA) synthesis [10].

Jasmonic acid alters physiological and developmental processes, such as plant growth and development, senescence, fruit ripening and also acts as a signal molecule in plant defense system responding to various biotic and abiotic stresses [11]. JA concentration in plant tissues depends on environmental stimuli, plant developmental stage and physiological conditions [11]. Octadecanoid pathway is initiated by linoleate: oxygen 13-oxidoreductase (LOX) (EC 1.13.11.12) (Figure 1) by its dioxygenase activity on LNA to produce hydroperoxy LNA which is followed by the action of allene oxide synthase and allene oxide cyclase [10]. There are evidences to show that low temperature increases the jasmonic acid content in spinach which further increased the levels of cytosolic $\mathrm{ca}^{2+}$ and abscisic acid [12]. Cold-induced accumulation of jasmonic acid prevents lipid peroxidation and increases cold resistance in apples[13]. Lipid peroxidation is a mechanism of cellular injury in plants. Hence, malondialdehyde the end product of lipid peroxidation was used to determine the oxidative stress in tissues of pea plants [14]. Abscisic acid (ABA) plays a major role in plant defence against

Copyright: (c) The authors. This article is open access and licensed under the terms of the Creative Commons Attribution License (http://creativecommons.org/licenses/by/4.0/) which permits unrestricted, use, distribution and reproduction in any medium, or format for any purpose, even commercially provided the work is properly cited. Attribution - You must give appropriate credit, provide a link to the license, and indicate if changes were made. 


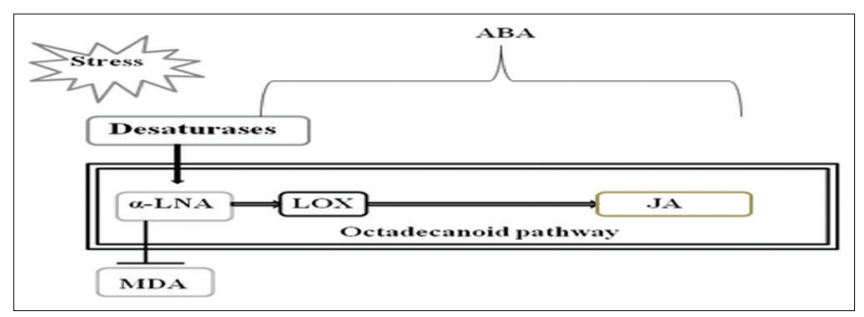

Figure 1: Schematic diagram showing the key components of the octadecanoid pathway. Key components of octadecanoid pathway involved in abiotic stresses. Omega 3 fatty acid desaturases, $\alpha$-linolenic acid(LNA), lipoxygenase(LOX), malondialdehyde(MDA), jasmonic acid $(\mathrm{JA})$, absciasic acid(ABA)

abiotic stress. ABA accumulation helps to maintain the water balance and cellular dehydration in plants [15]. An antagonistic interaction was observed between jasmonic and abscisic acid signalling pathways in cold and drought stresses. Omega 3 desaturase genes are known to be induced by a wide array of stresses such as cold ,drought, heat, salinity, disease etc which activates the octadecanoid pathway for significant accumulation of jasmonic acid[16,17]. Based on this hypothesis the expression of omega 3 fatty acid desaturases, quantification of jasmonic and abscisic acid, determination of lipoxygenase and malondialdehyde assays and fatty acid content under abiotic stresses were assessed to understand LNA accumulation in sesame.

\section{MATERIALS AND METHODS}

\section{Plant Material}

Sesame seed Variety TMV 7 was obtained from the Oil seeds Research Station (ORS), Tindivanam of Tamilnadu Agricultural University, Tamil Nadu, India. The seeds were surface sterilized and placed in petriplates containing half MS (Murashige and Skoog) basal medium for growth and maintained at $25 \pm 2^{\circ} \mathrm{C}$ with $16 \mathrm{~h}$ cool-white fluorescent lights and $8 \mathrm{~h}$ darkness. One month old seedlings were subjected to various abiotic stresses such as cold, drought, salinity and heat. Cold treatment was given by exposing the petriplates containing seedlings at $4^{\circ} \mathrm{C}$ for $5 \mathrm{~h} \mathrm{[18].} \mathrm{For} \mathrm{salinity} \mathrm{and} \mathrm{drought} \mathrm{stress} \mathrm{treatment,} \mathrm{the} \mathrm{seedlings}$ from plate were uprooted and kept in $200 \mathrm{mM} \mathrm{NaCl}$ solution and in 20\% PEG 6000 for 5h respectively [19]. For heat stress, the seedlings were removed from plate and exposed to $39^{\circ} \mathrm{C}$ for $5 \mathrm{~h} \mathrm{[9].Unstressed} \mathrm{sesame} \mathrm{seedlings} \mathrm{were} \mathrm{used} \mathrm{as} \mathrm{control.}$

\section{Quantitative RT-PCR Analysis}

Omega 3 desaturase genes from draft genome sequence of Sesamum indicum were retrieved by performing comparative analysis. Microsomal fatty acid desaturase (FAD3), chloroplast fatty acid desaturases (FAD7 and FAD8) were identified. Gene specific primers were designed for analysis of mRNA levels of the above mentioned genes and ubiquitin gene was used as housekeeping gene. Isolation of Total RNA from various stressed sesame seedlings using RNAeasy plant mini kit (Qiagen, Netherlands). First strand cDNA synthesis was performed using Revert Aid First Strand cDNA synthesis kit (Thermo Scientific, USA). Primers used in this study are listed in Table 1. SYBR
Table 1: List of primers used in quantitative real time PCR analysis

\begin{tabular}{llll}
\hline S.N0. & PRIMER SEQUENCE & ACCESSION N0. \\
\hline 1 & UBQ F & 5'CACCAAGCCGAAGAA & JP631638 \\
& & GATCAAG 3' & \\
2 & UBQ R & 5'CCTCAGCCTCTGCACCTTTC 3' $^{\prime}$ & \\
3 & FAD7 F & 5'GGCGGAGAAGAATTTGACCC3' & U25817.1 \\
4 & FAD7 R & 5'ACAAAGGCCAAACAACCCAA3' & \\
5 & FAD8 F & 5'TGTTCTGGGCTCTCTTCGTT3' & XM_011078407.1 \\
6 & FAD8 R & 5'AAGGGTGCCAGGATTCATCA3' & \\
7 & FAD3 F & 5'CCGCCATTCCTCCTTCCTAA3' & XM_011082487.1 \\
8 & FAD3 R & 5'GATCCTTCACCCAGCAATGC3' & \\
\hline
\end{tabular}

Premix Ex Taq II (Tli RNaseH Plus) (Takara, japan) and CFX96 Real-Time PCR detection system (Bio-Rad, USA) was used for detecting omega 3 desaturase genes. The amplification condition used was: $95^{\circ} \mathrm{C}$ for $30 \mathrm{sec}$ and 40 cycles of $95^{\circ} \mathrm{C}$ for $5 \mathrm{sec}$ and $60^{\circ} \mathrm{C}$ for $30 \mathrm{sec}$ followed by $95^{\circ} \mathrm{C}$ for $15 \mathrm{sec}, 60^{\circ} \mathrm{C}$ for $1 \mathrm{~min}, 95^{\circ} \mathrm{C}$ for 15 sec(melt curve).All reactions were performed for three replicates. The relative gene expression was calculated using $2^{-\Delta \Delta C t}$ method.

\section{LNA Quantification}

One gram of treated seedlings and control powdered using liquid nitrogen were taken for lipid extraction. Lipids were extracted with Chloroform: methanol (2:1) as previously described by [20]. $50 \mathrm{mg}$ of lipids were taken in Teflon-lined screw capped glass tube add $\mathrm{lml}$ of freshly prepared $2.5 \% \mathrm{H}_{2} \mathrm{SO}_{4}$ in methanol. They were incubated at $80^{\circ} \mathrm{C}$ for 2 hours followed by the addition of $500 \mu \mathrm{l}$ of hexane and $1.5 \mathrm{ml}$ of $0.9 \% \mathrm{NaCl}$ to extract fatty acid methyl esters (FAME).The contents were mixed thoroughly and centrifuged at 1000rpm for 3 mins to facilitate phase separation. The upper phase was transferred to new tube and concentrated with nitrogen stream and dissolved in $100 \mu$ l of hexane [21].

\section{Gas Chromatography Analysis}

FAME were analysed on Systronics Gas Chromatography instrument equipped with capillary column of $30 \mathrm{~m} \times 0.3 \mathrm{~mm}$ internal diameter and Flame ionization detector. The oven was programmed to hold the temperature at $100^{\circ} \mathrm{C}$ and increased up to $200^{\circ} \mathrm{C} / 5 \mathrm{~min}$. Nitrogen was used as a carrier gas. The Flame ionization detector and injector temperature was maintained at $260^{\circ} \mathrm{C}$. The peak was identified by comparison with the retention time of a commercial standard mixture of FAME (Supelco 37-component FAME mix Sigma, USA). Lipid analysis was performed independently for three times. Values were reported as average and standard deviation $(n=3)$.

\section{Quantification of Lipoxygenase Activity}

Powdered plant samples were homogenised in 3 volumes of $0.05 \mathrm{molL}^{-1}$ sodium phosphate buffer $\mathrm{pH} 6.5$ according to [22] The supernatant collected after centrifugation at $5000 \mathrm{rpm}$ for 30 mins at $4^{\circ} \mathrm{C}$ was filtered using $0.22 \mu \mathrm{m}$ filter (Pall Life Sciences). Protein was estimated with BSA as standards using bicinchoninic acid assay [23]. Lipoxygenase activity was assayed as per the method of Pinto et al., 2007. Known amounts of samples were 
incubated with the freshly prepared assay solution at $60^{\circ} \mathrm{C}$ for $15 \mathrm{mins}$. The test solutions were then cooled to room temperature and absorbance were measured at $562 \mathrm{~nm}$. LOX activity in the plant extracts was measured with LA as substrate. Optimum $\mathrm{pH}$ was determined by using a buffer system with the $\mathrm{pH}$ range of 1.5-10 at a final concentration of $0.05 \mathrm{molL}^{-1}$. The increase in absorbance due to hydroperoxide formation at $234 \mathrm{~nm}$ was measured for the samples in triplicates and the rate of reaction was measured using extinction coefficient $25 \mathrm{mM}^{-1} \mathrm{~cm}^{-1}$ [24].

\section{Quantification of MDA}

Malondialdehyde was measured under various abiotic stresses. The extraction procedure was carried as per [25].The optical density of the extract was measured by subtracting the nonspecific absorbance at $600 \mathrm{~nm}$ from the absorbance at $532 \mathrm{~nm}$ and using an extinction coefficient of $155 \mathrm{mM}^{-1} \mathrm{~cm}^{-1}$ [26].

\section{Quantification of JA}

Jasmonic acid was extracted using the protocol followed by [27]. The final extract was filtered with a $0.22 \mu \mathrm{m}$ filter and stored at $4^{\circ} \mathrm{C}$. Quantification of JA was performed using Agilent HPLC system with $\mathrm{C} 18$ reverse phase column. Standard JA (Himedia, Mumbai) was used to optimize the conditions. Accordingly, detection wavelength of $195 \mathrm{~nm}$, temperature of $35^{\circ} \mathrm{C}$ and mobile phase of acetonitrile/water $(70 / 30, \mathrm{v} / \mathrm{v})$ with a flow rate of $0.75 \mathrm{ml} / \mathrm{min}$ were used to quantify the samples.

\section{Quantification of ABA}

Abscisic acid was extracted using the modified protocol of [28]. A rotary flash evaporator was used and then the final residue obtained after evaporation was redissolved in $1 \mathrm{ml}$ of acetonitrile and stored at $4^{\circ} \mathrm{C}$. ABA (Duchefa) was diluted and standard concentrations were used to standardise the HPLC conditions in $\mathrm{Cl} 8$ column. The optimised conditions of 254nm wavelength and mobile phase of acetonitrile/acetic acid/water (60/0.04/39.96, v/v/v) with a flow rate of $1 \mathrm{ml} / \mathrm{min}$ was used to quantify the samples.

\section{Statistical Analysis}

Statistical analysis was performed using one way ANOVA and Dunnett Multiple Comparisons Test using Graph Pad InStat3 software. Error bars representing the standard deviation values were obtained from the triplicates $(n=3)$. All the experiments were replicated three times both the stress experiments and measurements of various components.

\section{RESULTS}

Quantification of Key Components of Octadecanoid Pathway Under Various Abiotic Stresses in Sesame

\section{Expression of omega 3 desaturase genes at RNA level}

The amount of mRNA corresponding to omega 3 desaturase genes FAD3, FAD7 and FAD8 present in cold, drought, heat, salt stressed and control sesame seedlings were quantified by real time PCR. The results were shown in Figure 2. Except cold other stresses exhibited lower levels of expression in FAD3 and FAD8 compared to control seedlings. The level of FAD7 and FAD8 chloroplast desaturase genes were found to be 9-fold and 3-fold higher than control during cold stress. In drought stressed seedlings the levels of expression of all three desaturases were found to be equivalent or lesser than control. FAD7 expression was found to be higher than other desaturases in heat and salinity. Expression of FAD7 was found to be higher in all four stresses among the three desaturases.

\section{Omega 3 fatty acid content}

In order to correlate the desaturase activity with omega 3 fatty acid content in the stressed seedlings and control the LNA content were measured using GC. $\alpha$ - linolenic acid content was found to be higher in cold, drought and heat stresses (Figure 3) whereas it was lower under salt stress in comparison with control. Cold exhibited the maximum level of LNA (1.2 mole \%) followed by heat $(0.9$ mole $\%)$ and drought $(0.7$ mole $\%)$ respectively.

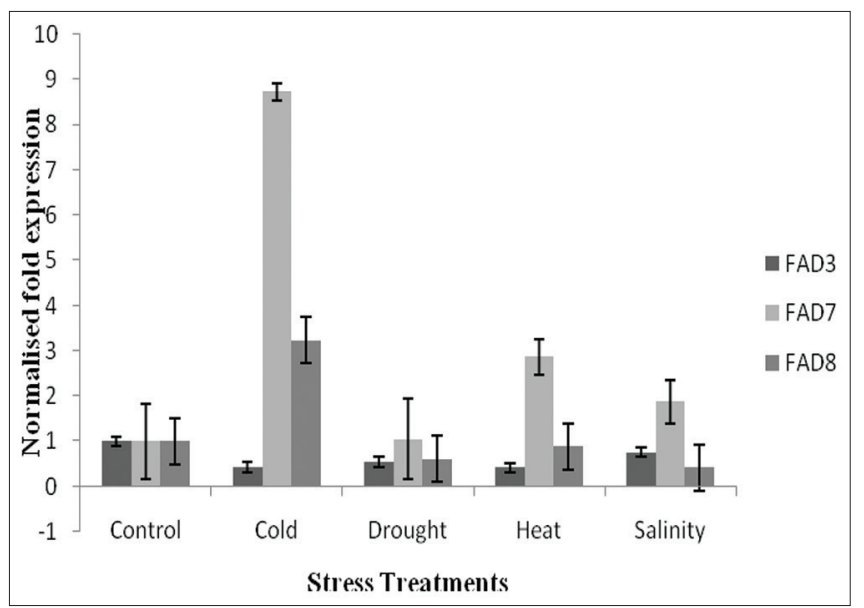

Figure 2: Quantitative RT-PCR analysis of $\omega 3$ desaturases of sesame. Expression levels of Omega 3 fatty acid desaturase genes in Sesamum indicum by Quantitative RT-PCR analysis.Normalization of gene expression against the housekeeping gene Ubiquitin. Data were obtained from three replicates (mean \pm SD)

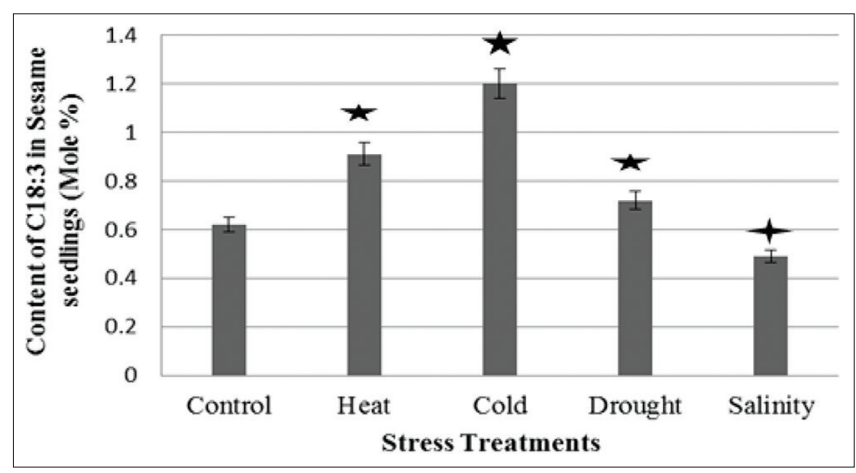

Figure 3: Omega 3 fatty acid analysis. Stress induced changes in C18:3 (omega3) content in sesame seedlings. Difference in the proportion of C18:3 content during stress treatments when compared to untreated control.Data obtained from three replicates (mean $\pm S D$ ). ( $\star$ ) denotes $\mathrm{P}<0.05$ difference in proportion changes of $\mathrm{C} 18: 3$ is significant when compared to untreated controls and $(+)$ denotes $P>0.05$ 


\section{Lipoxygenase activity}

Lipoxygenase is the first enzyme that utilizes LNA in the octadecanoid pathway. LOX activity in all four abiotic stressed seedlings is given in Figure 4. Heat stress exhibited significantly higher $(71.57 \mathrm{nM})$ amount of lipoxygenase activity compared with other stresses and control.LOX activity was found to be lesser in cold and drought stresses than control.

\section{Lipid peroxidation}

Malondialdehyde generated by lipid peroxidation is a measure of membrane damage. The malondialdehyde content was found to be higher in all stresses than control (Figure 5). The highest level of MDA was observed in heat stress (427.9nmol/gFW). Among all stresses cold stress exhibited least (176.6 nmol/gFW) MDA content.

\section{Jasmonic acid content}

Jasmonic acid is an important plant hormone involved in abiotic stress tolerance. It is also a key signalling molecule and end product of octadecanoid pathway. Jasmonic acid content was quantified to analyse its role in sesame seedlings subjected to various abiotic stresses. Jasmonic acid content was found to be higher than control in all stresses except heat stress (Figure 6). Salinity exhibited the maximum level of jasmonic acid accumulation $(2.12 \mu \mathrm{g} / \mathrm{gFW})$ followed by drought $(1.10 \mu \mathrm{g} / \mathrm{gFW})$ and cold $(1.07 \mu \mathrm{g} / \mathrm{gFW})$.

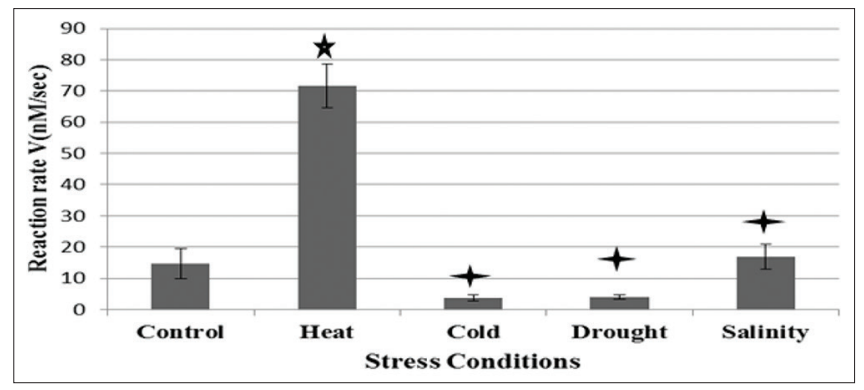

Figure 4: Estimation of LOX activity in sesame seedlings. Effect of LOX activity on abiotic stresses of sesame. Each value represents the mean of three independent measurements $\pm S D$. ( $\star$ ) sign indicates significant $\mathrm{P}<0.05$ and $(+) \mathrm{P}>0.05$

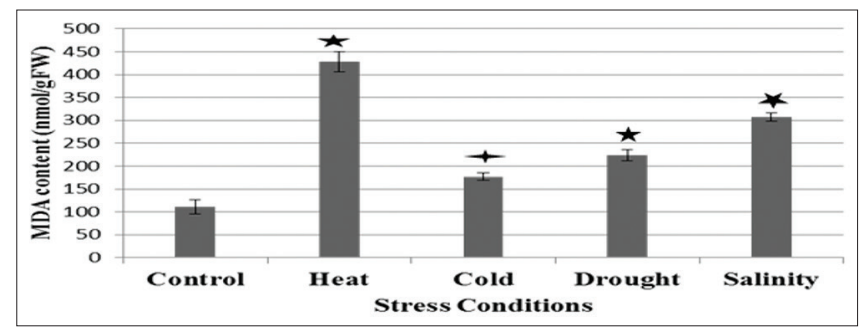

Figure 5: Estimation of MDA content in sesame seedlings. Determination of MDA content in different abiotic stressed sesame seedlings compared to unstressed seedlings $(\star)$ denotes $\mathrm{P}<0.05$ $(t)$ denotes $P<0.01$. Error bars represented \pm by means of standard deviation

\section{Abscisic acid content}

Abscisic acid is an octadecanoid pathway independent plant hormone that influences abiotic stress tolerance. The levels of $\mathrm{ABA}$ were measured to determine its role with reference to jasmonic acid in abiotic stressed sesame seedlings. Abscisic acid levels were higher than control in all stresses except salinity equivalent to control (Figure 7). Drought stress showed maximum ABA levels $(7.44 \mu \mathrm{g} / \mathrm{gFW})$ followed by heat $(6.3 \mu \mathrm{g} /$ gFW) and cold $(6.05 \mu \mathrm{g} / \mathrm{gFW})$ also showed increased ABA levels. Control $(5 \mu \mathrm{g} / \mathrm{gFW})$ and salinity $(4.84 \mu \mathrm{g} / \mathrm{gFW})$ exhibited almost equivalent levels of ABA.

\section{DISCUSSION}

\section{Fatty Acid Desaturation Affects Membrane Fluidity}

Lipid peroxidation is a measure of membrane damage which generates malondialdehyde in octadecanoid pathway using

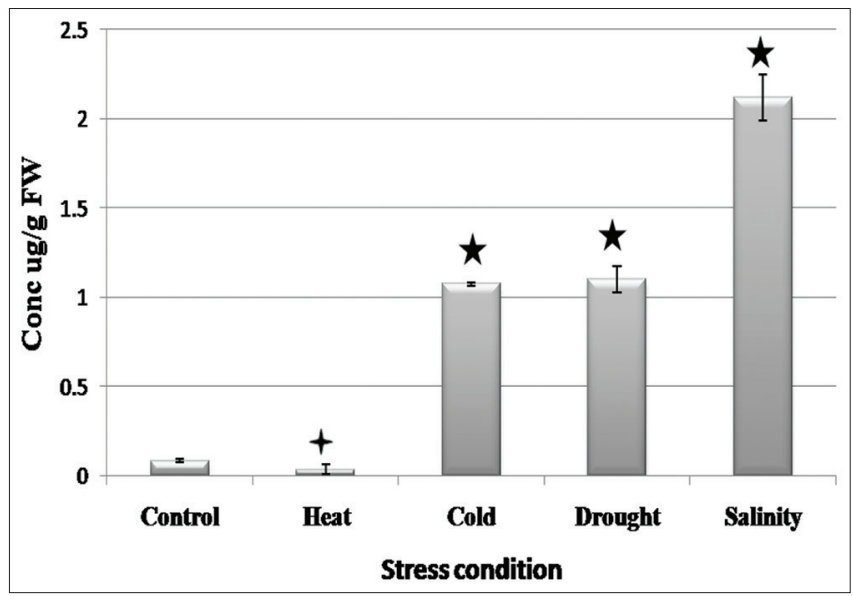

Figure 6: Determination of Jasmonic acid in sesame. Quantification of jasmonic acid levels in abiotic stresses of sesame. $(\star)$ denotes $P<0.01$ and $(t)$ denotes $P>0.05$. Error bars represented \pm by means of standard deviation

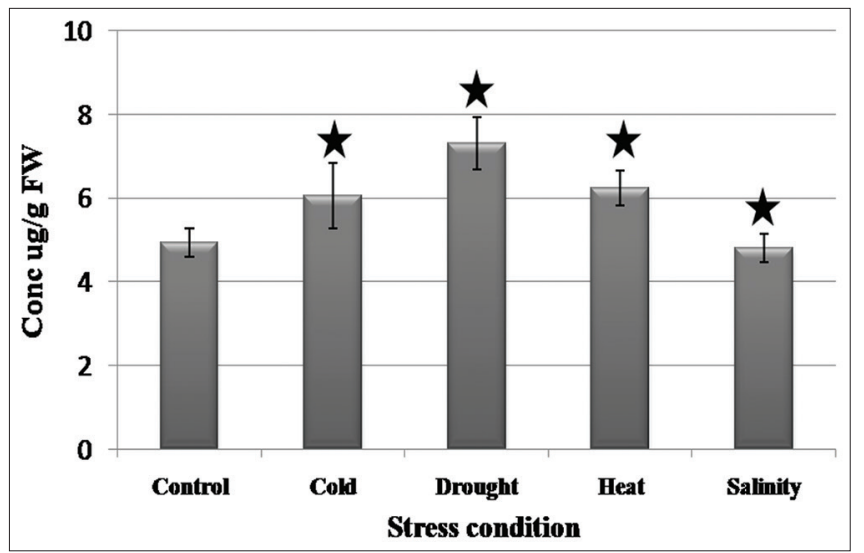

Figure 7: Determination of ABA content in sesame seedlings. Quantification of abscisic acid content signaling molecule under various abiotic stresses of sesame. $(\star)$ denotes $P<0.01$ significantly higher when compared to unstressed controls. Data obtained from three independent replicates (mean $\pm \mathrm{SD}$ ) 
LNA (Figure 1). In this study, MDA content of sesame seedlings subjected to various abiotic stresses were measured (Figure 5).Though all abiotic stresses showed higher MDA than control cold exhibited the lowest level indicating least damage. This suggests a key role of octadecanoid pathway in cold acclimation. Hence, desaturase mRNA levels and LNA accumulation (Figure 2 and 3 ) is expected to be higher in cold condition. The results of the present study clearly correlate to this hypothesis in sesame. In soybean GmFAD3A, GmFAD3B and GmFAD3C mRNA levels and omega 3 (18:3) content were found to be higher in cool $\left(20^{\circ} \mathrm{C}\right)$ than warm $\left(30^{\circ} \mathrm{C}\right)$ growing conditions [29]. Short term transient responses of cold stress in olive leaves were found to increase the expression of fatty acid desaturases [30]. In the present study among the three desaturases analyzed FAD7 and FAD8 are present in chloroplast whereas FAD3 is a microsomal desaturase. Among them, the expression of microsomal FAD3 was found to be the least in all four stresses and they were found to be lesser than control seedlings. This implies the role of FAD3 in abiotic stress tolerance is minimal. In Arabidopsis, higher chloroplast fatty acid desaturase mRNA levels (FAD7 and FAD8) and LNA content were correlated to chilling treatment $\left(4^{\circ} \mathrm{C}\right)$ and was also confirmed by generating octadecanoid pathway mutants and wild type plants [31]. Both chloroplast FAD7 and microsomal FAD3 in tomato also exhibited higher mRNA levels in cold stress [32]. In this investigation, the cold stress for sesame seedlings were also given at $4^{\circ} \mathrm{C}$ and the mRNA levels of FAD7 and FAD8 were found to be higher than the other stresses. Similarly, LNA content was also found to be higher in cold stressed seedlings. The above observations suggest an important role of chloroplast fatty acid desaturases in adaptation to cold in sesame.

In plants, hydro peroxides of LNA is utilised by both LOX and MDA which influences the membrane integrity. LOX activity and MDA content were found to be maximal at heat stress (Figures 4 and 5) whereas cold, drought and salt exhibited similar pattern of activity and content. Higher LOX activity and MDA content might lead to temperature associated oxidative damage in membranes. In our study the LOX activity and MDA content is found to be minimal in cold stressed seedlings when compared to control and other stresses (Figure 4 and 5). This observation suggests that among the four abiotic stresses cold stress is least influenced by the lipid peroxidation. The lower lipoxygenase activity shows lesser membrane damage or better acclimation. Hence, fatty acid desaturation is expected to be higher in cold condition. Accordingly, higher accumulation of LNA was found in cold stressed seedlings (Figure 3). Similarly, in wheat decline in MDA levels with higher cold-acclimation with increasing levels of LNA (18:3) were observed in Norstar and Gerdish cultivars [33].

\section{Role of Jasmonic Acid and Abscisic Acid in Abiotic Stresses}

Jasmonic acid is the end product of octadecanoid pathway and influences various physiological processes such as vegetative growth and development, cell cycle regulation, fruit ripening and senescence [34,35]. Besides, jasmonic acid also play prominent role in coordinating defence induced responses leading to increased stress tolerance in plants [36]. Jasmonic acid and abscisic acid content were found to be significantly higher in salinity, drought and cold stressed sesame seedlings (Figure 6 and 7). The above observation suggests a similar role of these signalling molecules in these abiotic stresses. There are contradicting reports on the role of jasmonic acid and salt tolerance [37]. It is also suggested not just the level of JA decides the kind of response to salinity but their timing and control is more important [38]. Involvement of both these signalling molecules in abiotic stresses was well documented by huge number of supporting reports and reviewed by [39]. However, the exact mechanism of their roles in abiotic stress tolerance is not understood completely. Differences in results and conclusion drawn could be due to variations in experimental conditions, developmental stages, tissues and degree of stress imposed [40]. In this study, salinity stress exhibited maximum amount of jasmonic acid whereas drought exhibited maximum abscisic acid content. Jasmonic acid content was found to be minimal, even lesser than control in heat stressed seedlings whereas in cold it was moderately higher (Figure 6). In tobacco, during elevated temperature, the amount of trienoic acid is found to be lower and end product jasmonic acid of octadecanoid pathway is observed to be higher [41]. Contrarily in sesame, we observed the lowest amount of JA in heat stress whereas LNA content was higher than control. The possible reasons could be either the amount of heat stress given may not be equivalent $\left(39^{\circ} \mathrm{C} \mathrm{Vs} 30^{\circ} \mathrm{C}\right)$ or the tissues in which LNA were quantified (seedling Vs seed) are different.

Abscisic acid is another fast responding signalling molecule which triggers JA in abiotic stress [42]. Drought stress recorded maximum level of ABA accumulation (Figure 7) suggesting a key role of these signalling molecule. Similarly in rice and soybean higher abscisic acid levels were observed when plants were exposed to water deficit conditions [28, 43]. In this study, JA and ABA levels were found to be synergistically higher in cold and drought stresses. Among the abiotic stresses cold stress seems to be influenced by the octadecanoid pathway whereas abscisic acid could play a role in the other stresses in sesame. An ABA induced transient drought experiment in Arabidopsis has shown decrease in JA [44]. JA and ABA levels could be modulated by intracellular calcium levels, accumulation of phospholipases, osmolytes and antioxidants was reported by [45]. Hence there is a possibility of such modulation in sesame also.

\section{ACKNOWLEDGEMENT}

The authors thank the Department of Biotechnology, PSG College of Technology and Department of Science and Technology, Government of India for providing the funding and infrastructure. We thank Tamilnadu Agricultural University, Coimbatore for providing TMV-7 sesame seeds.

\section{REFERENCES}

1. Hiremath SC, Patil CG, Patil KB, Nagasampige MH. Genetic diversity of seed lipid content and fatty acid composition in some species of Sesamum L. (Pedaliaceae). African Journal of Biotechnology.2007;6 
(5):539-543.

2. Anilakumar KR, Pal A, Khanum F, Bawa AS. Nutritional, medicinal and industrial uses of sesame (Sesamum indicum L.) seeds- An overview. Agriculturae Conspectus Scientificus.2010; 75:159-168.

3. Reshma MV, Balachandran C, Arumughan C, Sunderasan A, Divya S, Shiny Thomas Saritha SS. Extraction, separation and characterisation of sesame oil lignan for nutraceutical applications. Food Chemistry.2010; 120:1041-1046.

4. Wu WH. The contents of lignans in commercial sesame oils of Taiwan and their changes during heating. Food Chemistry.2007:104: 34-344.

5. Iba K. Acclimative response to temperature stress in higher plants: approaches of gene engineering for temperature tolerance. Annual Review of Plant Biology.2002; 53: 225-245.

6. Collados R, Andreu V, Picorel R, Alfonso MA. Light-sensitive mechanism differently regulates transcription and transcript stability of $\omega 3$ fatty-acid desaturases (FAD3, FAD7 and FAD8) in soybean photosynthetic cell suspensions. FEBS Letters. 2006; 580: 49344940 .

7. Nishiuchi T, Hamada T, Kodama H, Iba K. Wounding changes the spatial expression pattern of the Arabidopsis plastid $\omega 3$ fatty-acid desaturase gene (FAD7) through different signal transduction pathways. The Plant Cell.1997; 9: 1701-171.

8. Matsuda O, Sakamoto H, Nakao Y, Oda K, Iba K. CTD phosphatases in the attenuation of wound-induced transcription of jasmonic acid biosynthetic gene in Arabidopsis. The Plant Journal.2009; 57:96-108.

9. Zhang Y, Mian MR, Chekhovskiy V, So S, Kupfer D, Lai H, Roe BA. Differential gene expression in Festuca under heat stress conditions. Journal of Experimental Botany. 2005; 56:897-907.

10. Creelman RA, Mullet JE. Biosynthesis and action of jasmonates in plants. Annual Review of Plant Biology. 1997; 48:355-381.

11. Wang $Y$, Mopper S, Hasenstein KH. Effects of salinity on endogenous ABA, IAA, JA, and SA in Iris hexagona. Journal of Chemical Ecology.2001;27:327-342.

12. Yu C, Wang HS, Yang S, Tang XF, Duan M, Meng, OW. Overexpression of endoplasmic reticulum omega-3 fatty acid desaturase gene improves chilling tolerance in tomato. Plant Physiology and Biochemistry.2009; 47:1102-1112.

13. Yoshikawa H, Honda C, Kondo S. Effect of low-temperature stress on abscisic acid, jasmonates, and polyamines in apples. Plant Growth Regulation.2007; 52:199-206

14. Moran JF, Becana M, Iturbe-Ormaetxe I, Frechilla S, Klucas RV, Aparicio-Tejo P. Drought induces oxidative stress in pea plants. Planta.1994; 194:346-352.

15. Cutler S.R, Rodriguez PL, Finkelstein RR, Abrams SR. Abscisic acid: emergence of a core signaling network. Annual Review of Plant Biology. 2010; 61:651-679

16. Tang SY, Guan RZ, Zhang HS, Huang J. Cloning and expression analysis of three cDNAs encoding omega-3 fatty acid desaturases from Descurainia Sophia. Biotechnology Letters.2007:29:1417-1424.

17. Torres-Franklina M, Repellina A, Huynha V, Arcy-Lameta A, ZuilyFodil Y, Pham-Thia A. Omega-3 fatty acid desaturase (FAD3, FAD7, FAD8) gene expression and linolenic acid content in cowpea leaves submitted to drought and after rehydration. Environmental and Experimental Botany.2009; 65:162-169.

18. Margutti MP, Reyna M, Meringer MV, Racagni GE, Villasuso AL. Lipid signalling mediated by PLD/PA modulates proline and H2O2 levels in barley seedlings exposed to short-and long-term chilling stress. Plant Physiology and Biochemistry. 2017;113:149-60.

19. Wei L, Miao H, Zhao R, Han X, Zhang T, Zhang H. Identification and testing of reference genes for Sesame gene expression analysis by quantitative real-time PCR. Planta .2013; 237:873-89.

20. Bligh EG, Dyer WS. A rapid method of total lipid extraction and purification. Canadian Journal of Biochemistry and Physiology. 1959; 37:911-917

21. Christie WW. Lipid analysis: Isolation, separation, identification and structural analysis of lipids. Oily press, Bridgewater, UK. 2003.

22. Ohta H, Ida S, Mikami B, Morita Y. Changes in lipoxygenase components of rice seedlings during germination. Plant and cell physiology.1986;27:911-918.

23. Smith P, Krohn RI, Hermanson GT, Mallia AK, Gartner FH, Provenzano M, Fujimoto EK, Goeke NM, Olson BJ, Klenk DC. Measurement of protein using bicinchoninic acid. Analytical biochemistry. 1985; 150:76-85.

24. Pinto MC, Tejeda A, Duque AL, Macías P. Determination of lipoxygenase activity in plant extracts using a modified ferrous oxidation-xylenol orange assay. Journal of Agricultural and Food Chemistry.2007; 55:5956-5959.

25. Dhindsa RS, Plumb-Dhindsa P, Thorpe TA. Leaf senescence: correlated with increased levels of membrane permeability and lipid peroxidation and decreased levels of superoxide dismutase and catalase. Journal of Experimental Botany. 1981; 32:93-101.

26. Gülen H, Cetinkaya $C$, Kadıoğlu M, Kesici M, Cansev A, Eriş A Peroxidase activity and lipid peroxidation in strawberry (Fragaria $X$ ananassa) plants under low temperature. Journal of Biological and Environmental sciences.2008; 2 (6):95-100.

27. Xiong XJ, Rao WB, Guo XF, Wang H, Zhang HS. Ultrasensitive determination of jasmonic acid in plant tissues using highperformance liquid chromatography with fluorescence detection. Journal of Agricultural and Food Chemistry. 2012;60:5107-11.

28. Ali K, Gujjar RS, Niwas R, Gopal M, Tyagi A. A rapid method for estimation of abscisic acid and characterization of ABA regulated gene in response to water deficit stress from rice. American Journal of Plant Physiology.2011; 6:144-156.

29. Byfield GE, Upchurch RG. Effect of temperature on microsomal omega-3 linoleate desaturase gene expression and linolenic acid content in developing soybean seeds. Crop Science. 2007;47:24452452

30. Leyva-Pérez MD, Valverde-Corredor A, Valderrama R, Jiménez-Ruiz J, Muñoz-Merida A, Trelles O, Barroso JB, Mercado-Blanco J, Luque F. Early and delayed long-term transcriptional changes and short-term transient responses during cold acclimation in olive leaves. DNA research. 2014:22(1):1-1.

31. Shi Y, An L, Li X, Huang C, Chen G. The octadecanoid signaling pathway participates in the chilling-induced transcription of $\omega$-3 fatty acid desaturases in Arabidopsis. Plant Physiology and Biochemistry. 2011: 49:208-215

32. Domínguez T, Hernández ML, Pennycooke JC, Jiménez P, MartínezRivas JM, Sanz C, Stockinger EJ, Sánchez-Serrano JJ, Sanmartín M. Increasing $\omega$-3 desaturase expression in tomato results in altered aroma profile and enhanced resistance to cold stress. Plant Physiology.2010;153:655-665

33. Nejadsadeghi L, Maali-Amiri R, Zeinali H, Ramezanpour S Sadeghzade B. Membrane fatty acid compositions and cold induced responses in tetraploid and hexaploid wheats. Molecular biology reports. 2015;42:363-372.

34. Zhang Y, Turner JG. Wound-induced endogenous jasmonates stunt plant growth by inhibiting mitosis. PLoS ONE. 2008;3:e3699.

35. Yoshida Y, Sano R, Wada T, Takabayashi J, Okada K. Jasmonic acid control of GLABRA3 links inducible defense and trichome patterning in Arabidopsis. Development.2009;136:1039-1048.

36. Sreenivasulu N, Harshavardhan VT, Govind G, Seiler C, Kohli A Contrapuntal role of ABA: does it mediate stress tolerance or plant growth retardation under long-term drought stress? Gene. 2012;506: 265-273.

37. Sharma M, Laxmi A. Jasmonates: emerging players in controlling temperature stress tolerance. Frontiers in plant science.2016; 6:1129.

38. Ismail A, Takeda S, Nick P. Life and death under salt stress: same players, different timing? Journal of Experimental Botany. 2014;65: 2963-2979.

39. Riemann M, Dhakarey R, Hazman M, Miro B, Kohli A. Exploring jasmonates in the hormonal network of drought and salinity responses. Frontiers in plant science. 2015; 6:1077

40. Rassaa N, Ben Haj Salah H, Latiri K. Thermal responses of Durum wheat triticum durum to early water stress. Consequence on leaf and flower development. Comptes Rendus Biologies.2008;331:363-371.

41. Upchurch RG. Fatty acid unsaturation, mobilization, and regulation in the response of plants to stress. Biotechnology Letters. 2008;30:967977.

42. Bennett TH, Flowers TJ, Bromham L. Repeated evolution of salttolerance in grasses. Biotechnology Letters. 2013; 9:20130029.

43. Hamayun M, Khan SA, Shinwari ZK, Khan AL, Ahmad N, Lee IJ. Effect of polyethylene glycol induced drought stress on physio-hormona attributes of soybean. Pakistan Journal of Botany. 2010; 42:977-986.

44. Yang C, Liu J, Dong X, Cai Z, Tian W, Wang X. Short-term and continuing stresses differentially interplay with multiple hormones to regulate plant survival and growth. Molecular plant. $2014 ; 7(5): 841-55$.

45. Gong Y, Rao L, Yu D. Abiotic stress in plants. Agricultural chemistry InTech, Rejika.2013. 